\title{
Caractérisation de la flore phytoplanctonique dans l'Aire Marine Protégée (AMP) de Bamboung et de deux sites environnants (Sénégal)
}

\author{
Ali Mohamed ABDOU SALAM ${ }^{1 *}$, Ngansoumana BA ${ }^{1}$, Ismaïla NDOUR ${ }^{2}$, Seyni SANE ${ }^{1}$, \\ Modou THIAW ${ }^{2}$, Ndongo DIOUF ${ }^{1}$, Jules DIOUF ${ }^{1}$, Djibril DIOP ${ }^{1}$, \\ Madiop GUEYE ${ }^{1}$, Patrice BREHMER ${ }^{3}$, Mame Samba MBAYE ${ }^{1}$, Aboubacry KANE ${ }^{1}$ et \\ Kandioura NOBA ${ }^{1}$ \\ ${ }^{1}$ Laboratoire Botanique Biodiversité, Faculté des Sciences et Techniques, Université Cheikh Anta Diop de \\ Dakar, B.P. 5005 Dakar-Fann, Sénégal. \\ ${ }^{2}$ Institut Sénégalais de Recherches Agricole (ISRA), Centre de Recherches Océanographiques de Dakar- \\ Thiaroye (CRODT), B.P. 2241 Dakar, Sénégal. \\ ${ }^{3}$ Institut de Recherches pour le Développement-France (IRD), UMR Lemar, B.P. 1386 Dakar, Sénégal. \\ *Auteur correspondant; E-mail : salamabdou913@gmail.com, alimohamed.abdousalam@ucad.edu.sn; \\ Tel: (+221) 771077121
}

\section{REMERCIEMENTS}

Cette étude a été réalisée dans le cadre du projet Jeune Equipe Associée à l'Institut de Recherche et de Développement du Laboratoire d'Ecologie Halieutique de l'Afrique de l'Ouest (JEA LEH AO). L'aboutissement de ce travail est le fruit d'un accompagnement de JEA et le département de Biologie Végétale de l'Université Cheikh Anta Diop de Dakar dans tous les sens, qui méritent notre profonde reconnaissance.

\section{RESUME}

Au Sénégal, peu d'études sur le plan planctonique ont été réalisées pour l'évaluation de l'efficacité des Aires Marines Protégées. Dans cette perspective, la caractérisation de la flore phytoplanctonique et sa distribution spatio-temporelle à l'intérieur et à l'extérieur de l'AMP de Bamboung a été réalisée en avril et en septembre 2016. Des prélèvements d'échantillons d'eau à l'aide d'un filet à plancton de $20 \mu \mathrm{m}$ de mailles ainsi que des mesures de paramètres physico-chimiques ont été faits dans chaque station. L'analyse des données environnementales a montré l'existence d'un effet intersaison avec des valeurs plus élevées en avril excepté la température. Par ailleurs, un effet d'inter-sites a été observé uniquement en septembre pour la température, la salinité, la conductivité et l'oxygène dissout. En termes de diversité phytoplanctonique, en avril, 49 espèces réparties dans 5 classes ont été identifiées dans l'AMP contre 47 espèces et 5 classes identifiées à Diomboss et 42 espèces et 5 classes à Sangako. En septembre, 57 espèces réparties dans 5 classes ont été répertoriées dans 1'AMP contre 54 espèces et 4 classes à Diomboss, et 51 espèces réparties dans 4 classes identifiées à Sangako. Les résultats seuls de la richesse spécifique du phytoplancton ne permettent pas d'apporter une contribution sur l'évaluation de l'efficacité de l'AMP de Bamboung.

(c) 2020 International Formulae Group. All rights reserved.

Mots clés : Phytoplancton, dynamique, composition taxonomique, AMP, Bamboung. 


\title{
Characterization of the phytoplankton flora in the Protected Marine Area (MPA) of Bamboung and two surrounding sites (Senegal)
}

\begin{abstract}
In Senegal, few planktonic data exist in the Marine Protected Areas (AMP). In this perspective, the characterization of phytoplankton flora and its spatial and temporal distribution within and outside the Bamboung AMP was carried out during in April and in september. Water samples were taken using a $20 \mu \mathrm{m}$ mesh plankton net and measurements of physico-chemical parameters were made at each station. The analysis of environmental data showed the existence of an inter-site effect during the two-month sampling period. In April, 49 species in 5 classes were identified in the AMP, compared to 47 species and 5 classes identified in Diomboss and 42 species and 5 classes in Sangako. In september, 57 species distributed in 5 classes were recorded in the AMP compared to 54 species distributed in 4 classes in Diomboss and 51 species and 4 classes identified in Sangako. The results of the specific richness of phytoplankton alone do not make it possible to make a contribution to the evaluation of the effectiveness of Bamboung's MPA.
\end{abstract}

(C) 2020 International Formulae Group. All rights reserved.

Keywords: Phytoplankton, dynamics, taxonomic composition, AMP; Bamboung.

\section{INTRODUCTION}

Durant ces dernières décennies les écosystèmes aquatiques ont subi de forts bouleversements. Différentes pressions d'ordre anthropiques ou naturelles, sont à l'origine de ces perturbations: pollution, changement climatique et surexploitation des ressources halieutiques (Beaugrand et al., 2010). Plusieurs conséquences ont découlé notamment le déséquilibre des écosystèmes marins entraînant la perte de la biodiversité. A cela s'ajoutent également des pertes économiques importantes car de nombreuses activités comme la pêche et le tourisme dépendent de cette biodiversité (Beaugrand et al., 2010).

Pour répondre à ces différentes pressions et améliorer les politiques de gestion des ressources marines, les Aires Marines Protégées (AMP) ont été promues à travers le monde comme solution pour la restauration et le maintien de la résilience des écosystèmes côtiers (Cazalet, 2004). Au Sénégal, suite aux engagements pris par les autorités lors du sommet mondial de Johannesburg sur le développement durable tenu en 2002, un décret présidentiel $\mathrm{N}^{\circ}$ 2004-1408 du 04 Novembre 2004 a permis la création de cinq AMP parmi lesquelles, l'AMP de Bamboung.
Depuis la mise en fonction de l'AMP de Bamboung en 2004, aucune étude sur le phytoplancton n'a été réalisée pour l'évaluation de son efficacité. Les quelques travaux réalisés ont porté sur : la comparaison des peuplements de poissons de l'AMP avec ceux de deux sites proches non protégées (Ecoutin et al., 2012) et sur l'évaluation de l'efficacité de l'AMP de Bamboung comme outils de restauration des ressources marines et gestion des stocks halieutiques (Sadio, 2015). Pourtant, Le phytoplancton occupe une place incontournable dans les milieux aquatiques. En effet, par la photosynthèse, il produit de l'oxygène nécessaire à la vie dans ces milieux. En plus, le phytoplancton constitue une de voies des réseaux trophiques aquatiques (Ndour et al., 2018). Par ailleurs, le phytoplancton représente un bon indicateur pour évaluer l'état de santé d'un écosystème aquatique.

De ce fait, pour pallier ce manque, la présente étude qui est une première traitant du phytoplancton dans une AMP au Sénégal, va contribuer à l'évaluation de l'efficacité de l'AMP de Bamboung et améliorer les connaissances sur la composition taxonomique $\mathrm{du}$ phytoplancton dans les eaux marines sénégalaises en général. D’une manière 
spécifique, il s'agit, dans un premier temps, de déterminer la composition taxonomique du phytoplancton au sein de l'AMP et dans ses environs durant les deux saisons puis évaluer sa variation dans le temps et dans l'espace.

\section{MATERIEL ET METHODES}

\section{Site d'étude}

L'AMP de Bamboung $\left(13^{\circ} 50 \mathrm{~N}-\right.$ $16^{\circ} 33 \mathrm{~W}$ ) est située dans la commune de Toubacouta (Département de Foundiougne), au sud de l'estuaire du Sine-Saloum, à $130 \mathrm{~km}$ au sud-Est de Dakar. Elle se trouve au cœur de la Réserve de Biosphère du Delta du Saloum avec une superficie de 6800 ha (De Morais et al., 2007). Elle est divisée en zone :

$\checkmark$ Avale (superficie $1 \mathrm{~km}^{2}$ ), une zone tampon localisée à l'embouchure du bolon au niveau de la rencontre des eaux du Diomboss et du Bamboung,

$\checkmark$ Intermédiaire ou centrale (superficie 3 $\mathrm{km}^{2}$ ), d'une longueur de $15 \mathrm{~km}$ à partir du confluent avec le Diomboss jusqu'à la vasière de la forêt de Kolé. Elle est soumise aux déversements souterrains de l'Ile de coco réduisant ainsi la salinité.

$\checkmark$ Amont ou terrestre qui se décompose en une zone de mangrove et une zone continentale. Elle couvre plus de la moitié de la surface de l'AMP.

Par leurs proximités par rapport à l'AMP, le bolong de Sangako et de Diomboss, non protégés, ont été choisis comme sites environnants.

\section{Collecte des données}

L'échantillonnage a été réalisé à l'intérieur de l'AMP de Bamboung et dans les deux sites environnants (Figure 1). Dans chacun de ces trois sites, 5 stations ont été échantillonnées pendant les deux campagnes, une en avril 2016 (saison froide) et l'autre en septembre 2016 (saison chaude). Les
Coordonnées géographiques de chaque station sont reportées au Tableau 1.

$\mathrm{Au}$ cours de chaque campagne, les échantillons du phytoplancton ont été collectés à l'aide d'un filet à plancton de $20 \mu \mathrm{m}$ de maille remorqué derrière une pirogue. Les échantillons ont ainsi été conservés dans des flacons de $100 \mathrm{ml}$ et fixées au formol de 5\%. La température, la salinité, l'oxygène et la conductivité ont été mesurés en surface et en profondeur dans toutes les stations durant les deux mois d'échantillonnage. La température, l'oxygène et la conductivité ont été mesurés à l'aide d'une sonde multi-paramètre YSI 85 et la salinité a été mesurée par un réfractomètre de précision à 1 unité de salinité.

\section{Traitement des échantillons}

$\mathrm{Au}$ laboratoire, les échantillons ont été observés au microscope optique (Motic) aux objectifs (x 40 et x 100). Des mesures de dimension et des photos ont été réalisées. L'identification des espèces a été faite au plus bas niveau taxonomique possible en se référant à certains travaux : Paulmier (1994); Compère et al. (2009); Gueye et al. 2014 ; Gueye (2016) et Konan et al. (2018).

\section{Analyses statistiques}

Les données environnementales ont été saisies sur Excel. Le logiciel R (R core team, 2018) a été utilisé pour analyser statistiquement et graphiquement les données. Des analyses de variances ANOVA (test paramétrique) et test de Kruskal-Wallis (test non paramétrique) ont été réalisés suivies du test de comparaison multiple (SNK.test) quand les effets étaient significatifs. Dans tous les cas, une différence est considérée significative lorsque la $\mathrm{p}$-value $\leq 0,05$. Une Analyse en Composante Principale (ACP) a été également effectuée grâce au package FactoMiner du logiciel R afin de caractériser l'environnement de l'AMP de Bamboung et ses environs. 

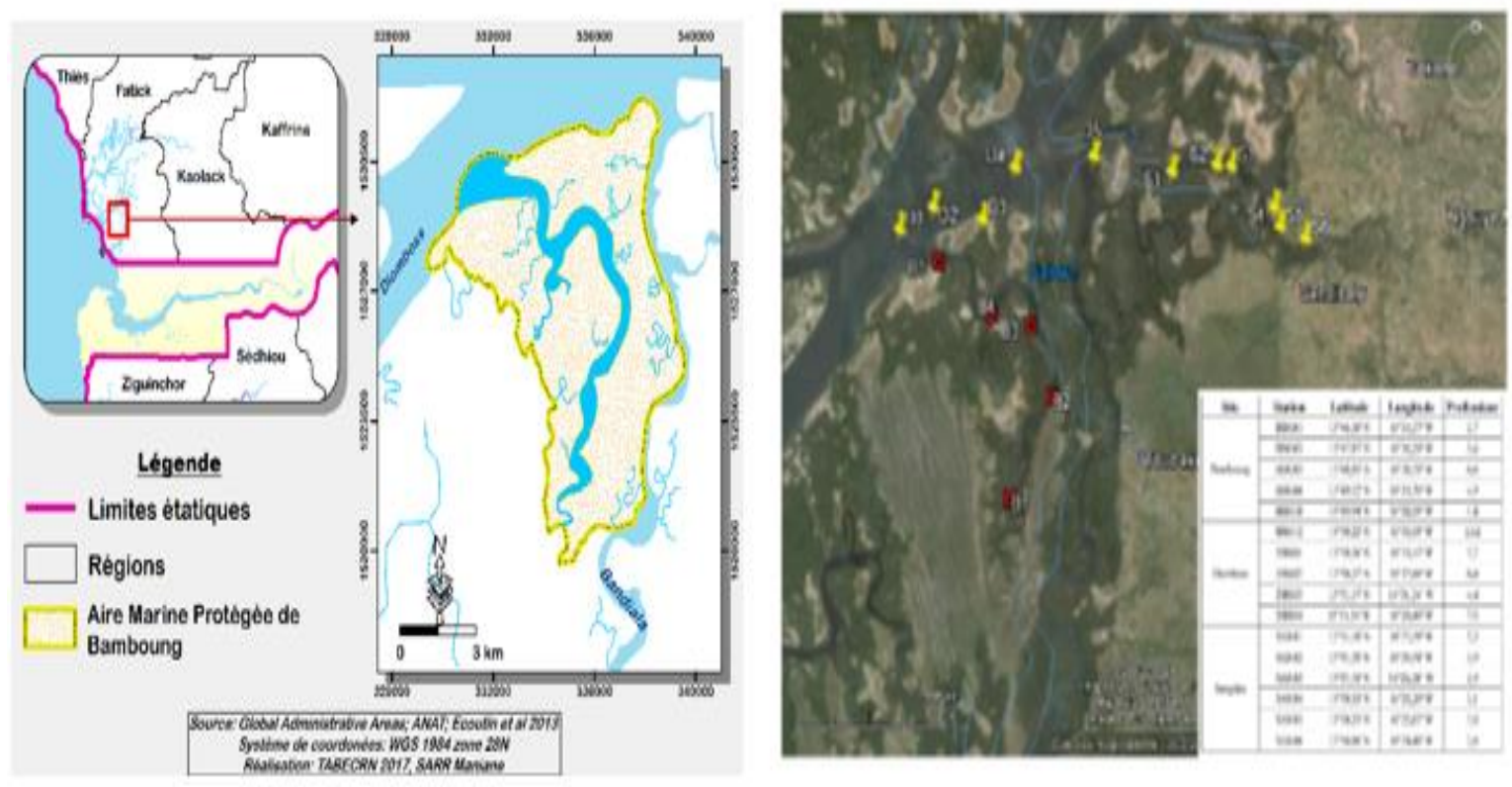

Figure 1: Localisation de l'Aire Marine Protégée de Bamboung et les sites environnants. $\mathrm{B}=$ AMP Bamboung, $\mathrm{D}=$ Diomboss et $\mathrm{S}=$ Sangako.

Tableau 1 : Référence des 3 sites et stations de l'AMP de Bamboung et ses environs.

\begin{tabular}{lllll}
\hline Sites & Stations & Latitude & Longitude & Profondeur (m) \\
\hline \multirow{5}{*}{ AMP Bamboung } & $\mathrm{BBG01}$ & $13^{\circ} 46,30^{\prime} \mathrm{N}$ & $16^{\circ} 31,27^{\prime} \mathrm{W}$ & 3,7 \\
\cline { 2 - 5 } & $\mathrm{BBG03}$ & $13^{\circ} 47,87^{\prime} \mathrm{N}$ & $16^{\circ} 30,29^{\prime} \mathrm{W}$ & 5,6 \\
\cline { 2 - 5 } & $\mathrm{BBG05}$ & $13^{\circ} 48,95^{\prime} \mathrm{N}$ & $16^{\circ} 30,79^{\prime} \mathrm{W}$ & 6,6 \\
\cline { 2 - 5 } & $\mathrm{BG08}$ & $13^{\circ} 49,12^{\prime} \mathrm{N}$ & $16^{\circ} 31,70^{\prime} \mathrm{W}$ & 4,9 \\
\cline { 2 - 5 } & $\mathrm{BG10}$ & $13^{\circ} 49,97^{\prime} \mathrm{N}$ & $16^{\circ} 32,95^{\prime} \mathrm{W}$ & 4,8 \\
\hline \multirow{5}{*}{ DIOMBOSS } & $\mathrm{BBG01}$ & $13^{\circ} 50,22^{\prime} \mathrm{N}$ & $16^{\circ} 33,93^{\prime} \mathrm{W}$ & 10 \\
\cline { 2 - 5 } & $\mathrm{DBS01}$ & $13^{\circ} 50,22^{\prime} \mathrm{N}$ & $16^{\circ} 33,93^{\prime} \mathrm{W}$ & 7,7 \\
\cline { 2 - 5 } & $\mathrm{DBS02}$ & $13^{\circ} 50,37^{\prime} \mathrm{N}$ & $16^{\circ} 32,00^{\prime} \mathrm{W}$ & 6,4 \\
\cline { 2 - 5 } & $\mathrm{DBS03}$ & $13^{\circ} 51,17^{\prime} \mathrm{N}$ & $16^{\circ} 31,24^{\prime} \mathrm{W}$ & 4,4 \\
\cline { 2 - 5 } & $\mathrm{DBS04}$ & $13^{\circ} 51,34^{\prime} \mathrm{N}$ & $16^{\circ} 29,40^{\prime} \mathrm{W}$ & 7 \\
\hline \multirow{5}{*}{ SANGAKO } & $\mathrm{SAN01}$ & $13^{\circ} 51,10^{\prime} \mathrm{N}$ & $16^{\circ} 27,59^{\prime} \mathrm{W}$ & 5,3 \\
\cline { 2 - 5 } & $\mathrm{SAN02}$ & $13^{\circ} 51,20^{\prime} \mathrm{N}$ & $16^{\circ} 26,54^{\prime} \mathrm{W}$ & 3,9 \\
\cline { 2 - 5 } & $\mathrm{SAN03}$ & $13^{\circ} 51,18^{\prime} \mathrm{N}$ & $16^{\circ} 26,20^{\prime} \mathrm{W}$ & 3,9 \\
\cline { 2 - 5 } & $\mathrm{SAN04}$ & $13^{\circ} 50,55^{\prime} \mathrm{N}$ & $16^{\circ} 25,20 \mathrm{~W}$ & 3,1 \\
\cline { 2 - 5 } & $\mathrm{SAN05}$ & $13^{\circ} 50,25^{\prime} \mathrm{N}$ & $16^{\circ} 25,07 ' \mathrm{~W}$ & 3 \\
\hline
\end{tabular}




\section{RESULTATS}

\section{Paramètres physico-chimiques}

La figure 2 représente la variation spatiale de la température, de la salinité, de l'oxygène et de la conductivité dans l'AMP de Bamboung et dans ses deux sites environnants en avril et septembre. Les résultats montrent une variation saisonnière avec des valeurs plus élevées en avril excepté la température. Toutefois, des réponses différentes ont été obtenues en comparant les trois sites. En effet, au mois d'avril, aucune variation significative des paramètres physico-chimiques entre l'AMP et Sangako n'a été notée (Figure 2.b). Par contre, la salinité $(p$-value $=0,03)$ et la conductivité $\quad(p$-value $=0,02)$ ont varié significativement entre l'AMP et Diomboss avec des valeurs plus élevées dans l'AMP (44,9 ppt et $70,4 \mathrm{~ms}$ contre 37,9 ppt et $57,7 \mathrm{~ms}$ ). Quant 'au mois de septembre (Figure 2.a), une variation significative a été observée pour tous les paramètres physico-chimiques entre les sites avec des températures et des pourcentages oxygènes moyennes plus élevées à Sangako $\left(33,3{ }^{\circ} \mathrm{C}\right.$ et $\left.86,5 \%\right)$ et à Diomboss $\left(31,9^{\circ} \mathrm{C}\right.$ et $75,4 \%)$ que dans l'AMP $\left(28,8^{\circ} \mathrm{C}\right.$ et $\left.74,7 \%\right)$. En revanche, la salinité a été plus élevée dans l'AMP (32,0 ppt) qu'à Diomboss $(30,0$ ppt) et à Sangako (27,8 ppt).

\section{Analyse des conditions environnementales}

Les relations entre les paramètres physico-chimiques ainsi que la typologie de différentes stations au mois d'avril sont illustrées par les graphes du cercle de corrélation (

Figure 3.a et 3.b). Les deux premières axes (axe 1 et axe 2) portent $96 \%$ de la variance total. L'axe 1 qui exprime $76 \%$ de la variance totale est un axe de gradient avec des fortes corrélations positives de l'ensemble des paramètres physico-chimiques dans les abscisses positives. L'axe 2 qui exprime 19\% de la variance totale permet d'isoler l'oxygène dans les ordonnées positives. Les deux axes mettent aussi en évidence une similarité entre l'AMP de Bamboung et ses deux sites environnants.

Durant le mois de septembre, les deux premières axes (axe 1 et axe 2 ) portent $95 \%$ de la variance total (Erreur ! Source du renvoi introuvable. 4.a et 4.b). L'axe $1(76 \%)$ est un axe qui oppose la température et l'oxygène dans les abscisses positives à la salinité dans les abscisses négatives. Il permet aussi de distinguer l'AMP de Bamboung dans les abscisses négatives et le bolong de Sangako dans les abscisses positives. L'axe 2 (19\%) est un axe de gradient de conductivité. Il permet de distinguer le bolong de Diomboss dans les abscisses positives et aux deux autres dans les abscisses négatives (Figure 4.b).

\section{Structure du phytoplancton dans I'AMP de Bamboung et ses environs}

Au total, 120 espèces (106 espèces de Diatomées, 8 espèces de Dinophycées et 6 espèces de Cyanophycées) réparties dans 31 familles et 5 classes ont été inventoriées. En avril, 49 espèces réparties dans 22 familles et 5 classes ont été identifiées dans l'AMP. Cette flore est caractérisée par la prédominance des Bacillariophyceae (32\%) et des Coscinodiscophyceae (32\%). Viennent ensuite les Cyanophyceae (14\%) et les Mediophyceae (13\%) (Figure 5.a). La classe des Dinophyceae (8\%) a été la moins diversifiée. Au sein de l'AMP, les espèces Anabaena $s p$ (Nostocaceae), Chaetoceros terres (Chaetocerataceae) et Thalassionema nitzschoides (Thalassionemataceae) avec toutes des fréquences relatives de $60 \%$, ont été les plus fréquentes.

Dans le Sangako, 42 espèces réparties dans 25 familles et 5 classes ont été identifiées. Comme dans l'AMP, les Bacillariophyceae (32\%) et les Coscinodiscophyceae (32\%) ont été les plus représentées. Viennent ensuite les Dinophyceae (16\%), les Mediophyceae (7\%) et les Cyanophyceae (5\%) (Figure 5.b). Les espèces Paralia sulcata (Paraliaceae) et Prorocentrum micans (Prorcentraceae) (Figure 6) ont été plus fréquentes avec respectivement $100 \%$ et $60 \%$ d'occurrence.

Dans le Diombos, 47 espèces réparties dans 22 familles et 5 classes ont été inventoriées. $\mathrm{La}$ classe des Coscinodiscophyceae (47\%) a été largement la plus diversifiée suivie par les Bacillariophyceae (19\%) et les Mediophyceae 
(19\%) et les Dinophyceae (12\%) (Figure 5.c). Les Cyanophyceae $(2 \%)$ ont été les moins prédominantes. Les espèces Paralia sulcata (100\%), Ceratium furca des Ceratiaceae (80\%), Prorocentrium micans $(80 \%)$ et Thalassiosira accentrica $(80 \%)$ de Thalassiosiraceae ont été les plus permanentes dans le Diomboss.

En septembre, 57 espèces reparties en 23 familles et 5 classes ont été identifiées au sein de l'AMP. La classe des Coscinodiscophyceae (47\%) a été largement la plus prédominante suivie par celle des Bacillariophyceae (33\%) (Figure 5.a). Les Dinophyceae $(9 \%)$, les Mediophyceae $(6 \%)$ et les Cyanophyceae $(5 \%)$ ont été les moins représentées. Les espèces Lithodesmium undilatum (100\%, Lithodesmiaceae) de, Bacillaria paradoxa $(80 \%)$ et B. paxilifora $(80 \%)$ des Bacillariaceae, Chaetoceros pseudocurvisetus $(80 \%)$ ont été les plus permanentes.
Dans les sites environnants, 51 espèces, 21 familles et 4 classes ont été identifiées à Sangako alors que 54 espèces, 20 familles et 4 classes ont été identifiées à Diomboss. Dans les deux cas, la classe des Coscinodiscophyceae a été largement plus représentée (53\% à Sangako et $57 \%$ à Diomboss) (Figure 5.b et 5.c) suivie par les Bacillariophyceae (29\% à Sangako et $28 \%$ à Diomboss). Les Mediophyceae (14\% à Sangako et $11 \%$ à Diomboss) et les Dinophyceae (4\% à Sangako et $4 \%$ à Diomboss) ont été les moins prédominantes. Quant'aux Cyanophyceae, elles n'ont pas été observées dans ces deux sites durant cette période. A Sangako, les espèces Lithodesmium undilatum (100\%), Navicula penata (Naviculaceae, 100\%), Bacillaria. paradoxa (80\%) Chaeteceros lauderii et C. teres (80\%) ont été les plus distribuées. Tandis qu'à Diomboss, Coscinodiscus oculis-irridis ( $80 \%$, Coscinodiscaceae) et Lithodesmium undulatum (80\%) ont été les plus répandues.
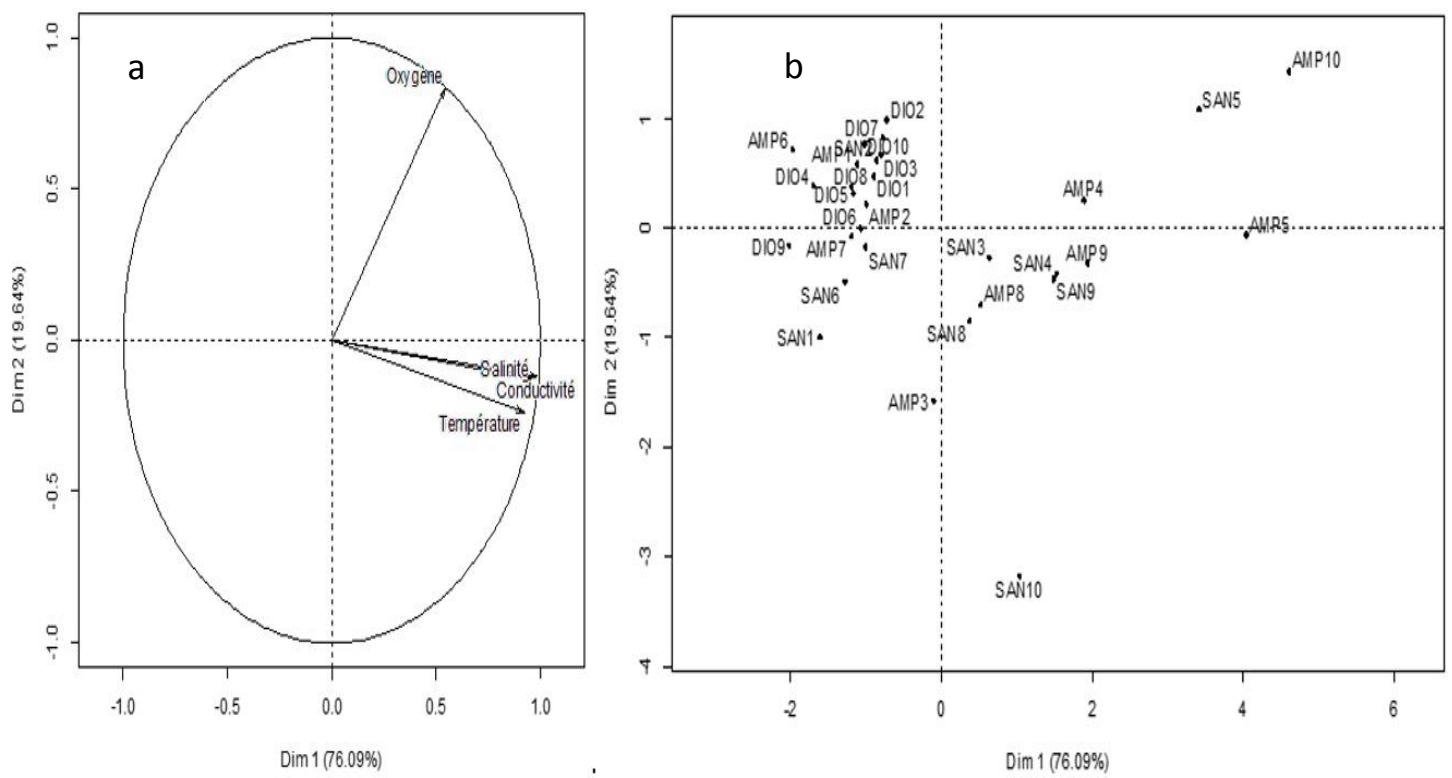

Figure 3: Analyse en composante principale des paramètres physico-chimiques des différentes stations de l'Aire Marine Protégée de Bamboung et ses environs au mois d'avril. a) Graphe des variables et b) Graphe des individus. AMP=AMP Bamboung, $\mathrm{SAN}=$ Sangako et DAM= Diomboss. 

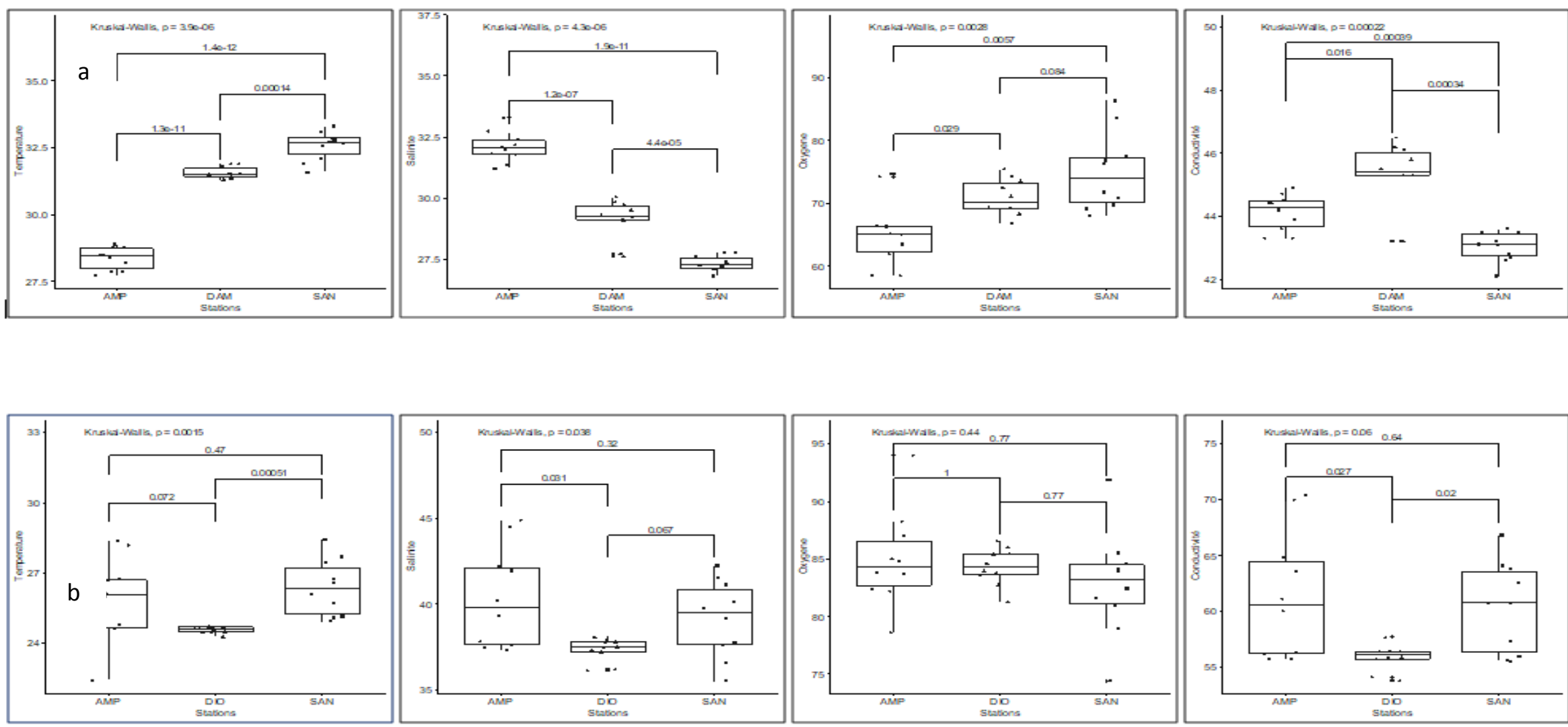

Figure 2 : Variation spatiale des paramètres physico-chimiques (température, salinité, oxygène et conductivité) dans l'AMP de Bamboung (AMP) et dans les bolons de Sangako (SAN) et de Diomboss (DIO) en Avrill (a) et septembre (b). 

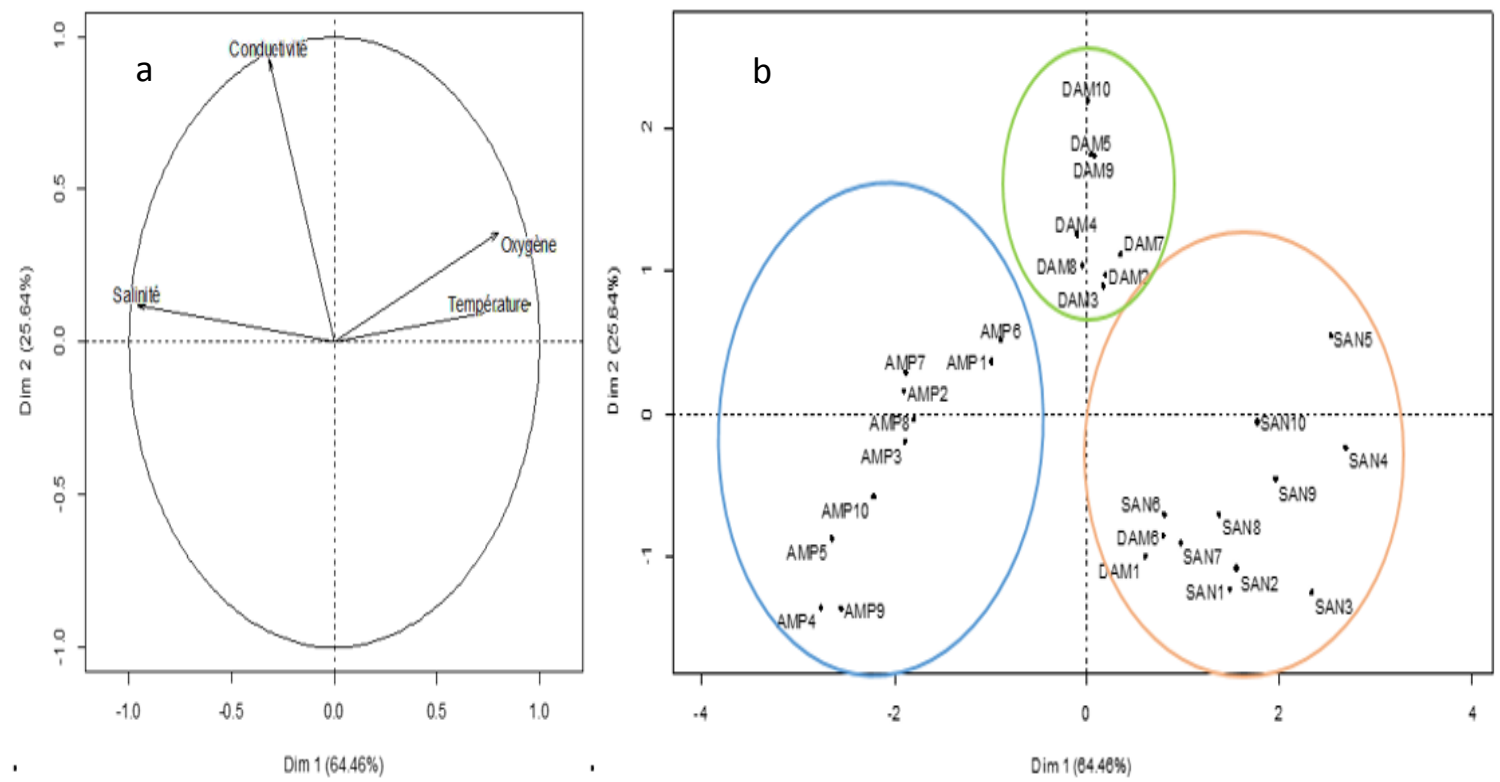

Figure 4: Analyse en composante principale des paramètres physico-chimiques des différentes stations au mois de septembre dans l'Aire Marine Protégée de Bamboung et ses environs. a) Graphe des variables et b) Graphe des individus. $\mathrm{AMP}=\mathrm{AMP}$ Bamboung, $\mathrm{SAN}=$ Sangako et $\mathrm{DAM}=$ Diomboss.
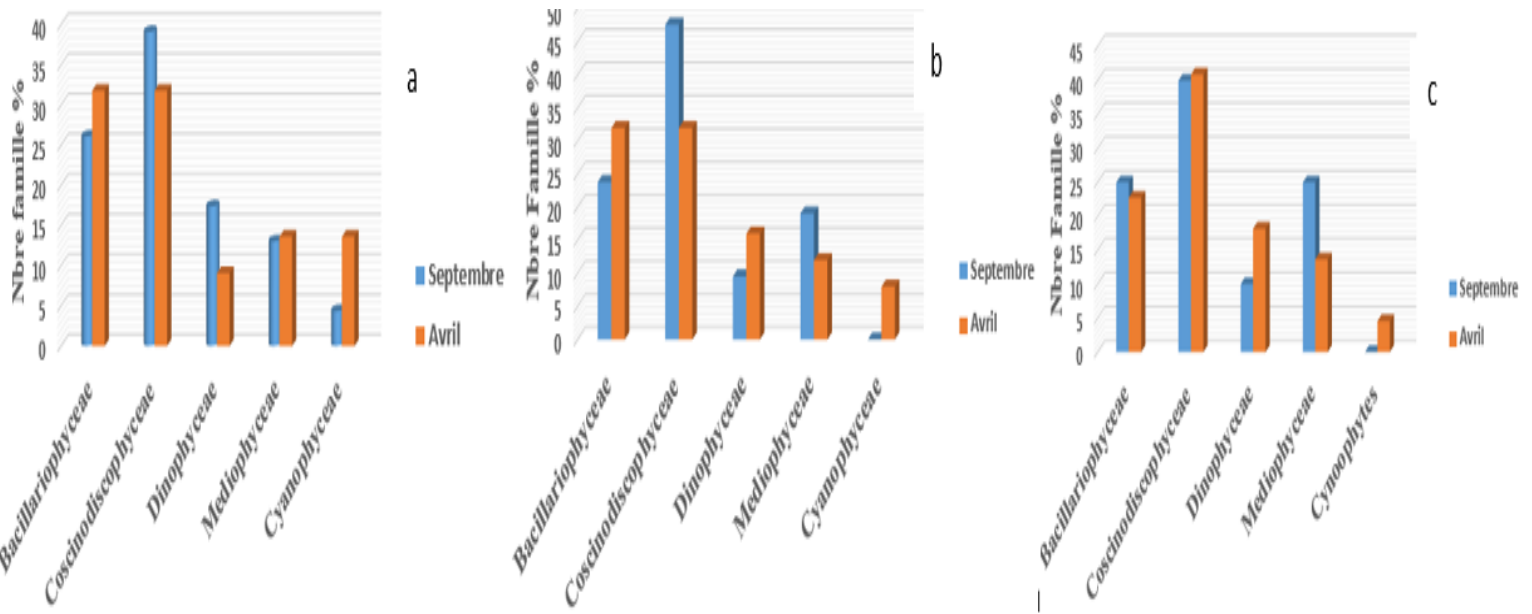

Figure 5 : Variation temporelle des classes du phytoplancton dans l'AMP de Bamboung (a) et dans les bolong de Sangako (b) et de Diomboss (c). 

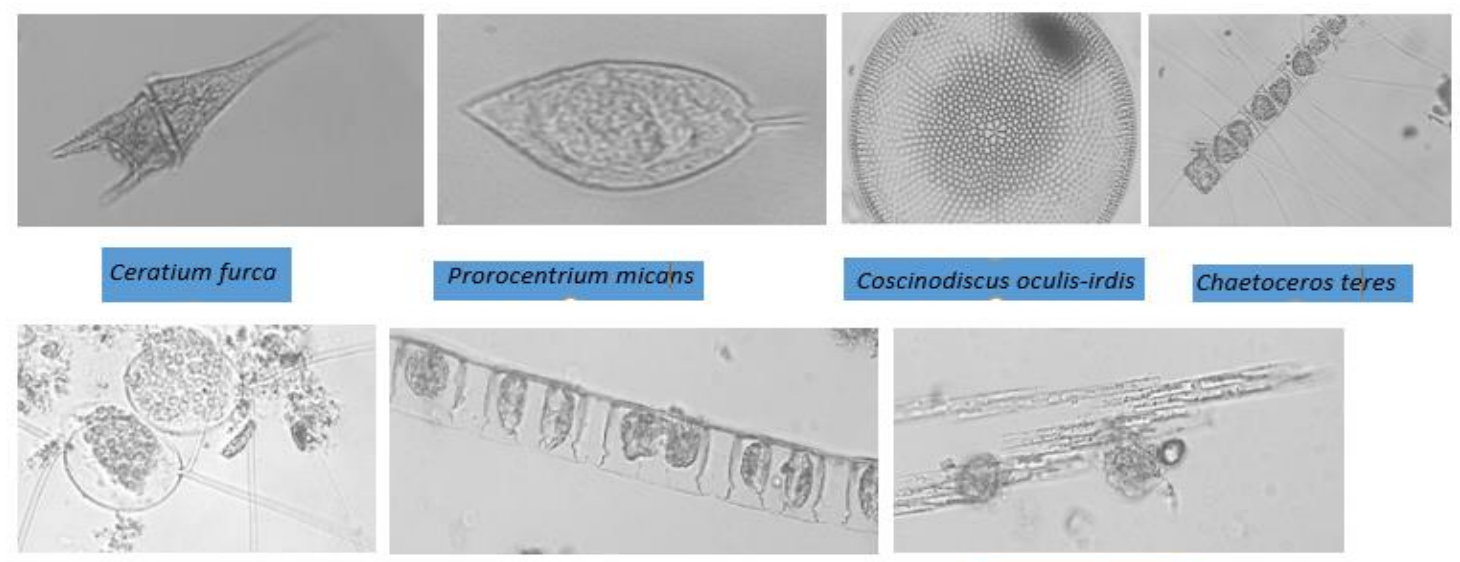

Coscinodiscus oculis-irdis
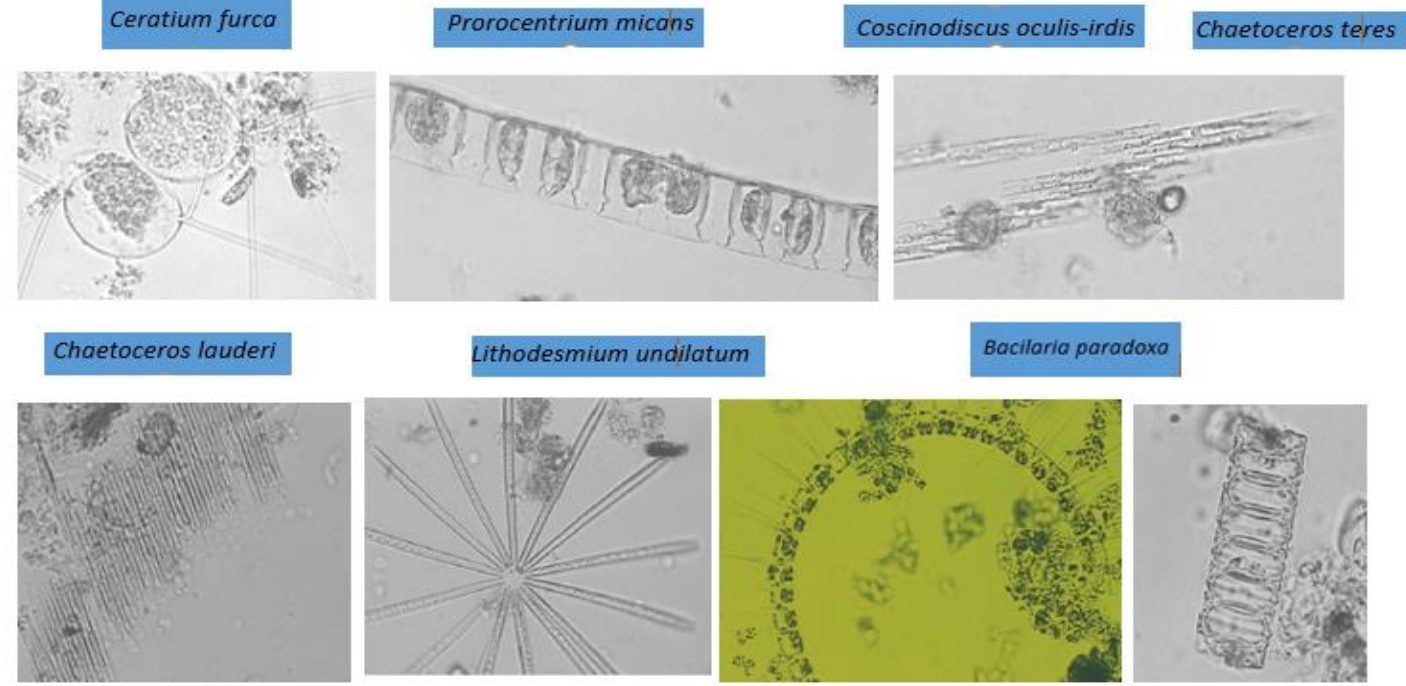

Bacilaria paxilifer

Thalassionema nitzschiodes
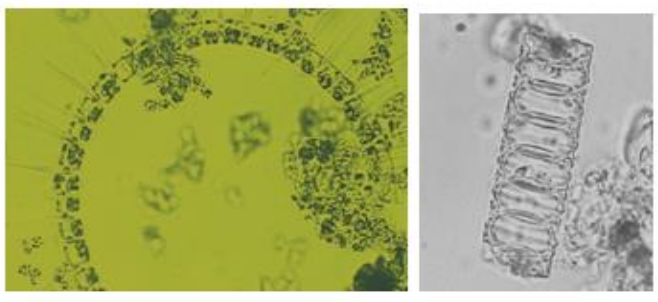

Chaetoceros pseudocurvisetus

Paralia sulcata

Figure 6 : Quelques espèces identifiées dans l'AMP de Bamboung et ses environs.

\section{DISCUSSION}

Durant l'échantillonnage, une similarité des différentes valeurs de paramètres physicochimiques entre surface et fond a été notée. Cela témoigne une absence de stratification verticale de la colonne d'eau et une homogénéité des différents sites. Ce fait pourrait s'expliquer par les faibles profondeurs et les courants marins moyens qui caractérisent les trois sites comme l'ont montré les travaux de De Morais et al. (2007). Par ailleurs, l'AMP a montré des différences par rapport à ses environs vis-à-vis de la température, la conductivité et la salinité malgré les courants et la proximité des stations uniquement en saison chaude. Cet effet d'inter-sites ne corrobore pas les résultats de Sadio (2015) et d'Ecoutin et al. (2012) qui ont souligné une absence d'une variation significative entre ses 3 sites indépendamment de la saison.

L'analyse des données biologiques a permis de recenser 120 taxons durant les deux mois d'étude. Cette valeur de richesse spécifique est supérieure à celle obtenue par Dia (1983) sur le long de la Petite côte sénégalaises (44 espèces). Par contre, elle est inférieure à celle trouvée par Gueye (2016) dans la presqu'île Du Cap Vert et de la Petite Côte (145 espèces). Ceci peut être expliqué par la nature et la taille d'échantillonnage. En effet, les travaux de Gueye s'intéressaient à la fois aux espèces planctoniques et benthiques.

Dans l'ensemble des trois sites et indépendamment de la saison, la flore a été caractérisée par la prédominance des Bacillariophytes, un groupe principalement marin, et la présence permanente des classes de Bacillariophyceae, de Coscinodiscophyceae, de Cyanophyceae, de Dinophyceae et de Mediophyceae. Cette dominance des Bacillariophytes, caractéristique des eaux marines, a été aussi soulignée par les travaux de Dia (1983), Ba et Noba (2011), et Gueye (2016). Elle peut s'expliquer d'une part par le 
fait que les Bacillariophytes sont beaucoup plus adaptées aux turbulences marines (Assougnon et al., 2017). D'autre part, les températures notées $\left(24\right.$ et $\left.30^{\circ} \mathrm{C}\right)$ sont aussi favorables aux Bacillariophytes (Jones et Poplwaski, 1998). La faible représentation des Cyanophytes et des Dinophytes peut être associée à leur besoin écologique. En effet, les travaux d'Atanle et al. (2013) ont montré que les Dinophytes prolifèrent davantage dans des salinités plus élevées. Ces auteurs ont souligné aussi le fait que les Dinophytes contrairement aux Bacillariophytes, sont beaucoup plus océaniques que côtières. Toutefois, la variation saisonnière et spatiale de paramètres environnementaux ne semble pas impacter la structure et la richesse spécifique du phytoplancton. Des études approfondies sur les éléments nutritifs, l'état thermique, le fonctionnement $\mathrm{du}$ plan d'eau semble nécessaire pour mieux interpréter le fonctionnement du phytoplancton dans cette zone.

\section{Conclusion}

L'analyse de données récoltées au cours de deux campagnes en avril et en septembre dans l'AMP de Bamboung et ses environs, constitue une première étape d'une caractérisation de la flore du phytoplancton dans ces zones de conservation au Sénégal. Il ressort de ses résultats une forte richesse spécifique en septembre qu'en avril. Il s'ensuit aussi une richesse spécifique légèrement élevée au sein de l'AMP indépendamment du mois. La comparaison de la diversité spécifique a montré qu'elle ne varie pas entre l'AMP et ses environs sauf en septembre où les Cyanophytes ne sont pas observées dans les sites environnants. Cependant, les résultats de la richesse spécifique seuls ne permettent pas d'apporter une précision sur l'efficacité de l'AMP de Bamboung.

\section{CONFLIT D'INTERETS}

Les auteurs déclarent qu'il n'y a aucun conflit d'intérêts pour cet article.

\section{CONTRIBUTIONS DES AUTEURS}

AS a collecté et observé les données biologiques. NB a dirigé l'identification des espèces et assuré notre encadrement. NI a dirigé les collectes des données et participé également dans l'encadrement. SS a orienté les analyses statistiques. TM, GM, DN, JD, DD, MMS, AK, BP et NK ont contribué à la correction de cet article.

\section{REFERENCES}

Assougnon DL, Agadjihouèdé H, Kokou K, Lalèyé AP. 2007. Caractérisation physico-chimique et diversité du peuplement phytoplanctonique des mares au sud de la réserve de faune de Togodo (sud-Togo). International Journal of Biological and Chemical Sciences, 11 (4) :

1920-1936.

DOI: $10.4314 / \mathrm{ijbcs.v11i4.40.}$

Atanle K, Baawa LM, Kokou K, Djaneye BG, Edorh MT. 2013. Distribution saisonnière $\mathrm{du}$ phytoplancton en fonction des caractéristiques physico-chimiques du Lac de Zowla (Lac Boko) dans le Sud-Est du Togo : cas de la petite saison sèche et de la grande saison sèche. Journal of Apllied Biosciences, 64: 4847-4857. DOI: $10.4314 /$ jab.v64i1.88474.

Ba AT, Noba K. 2011. Flore et biodiversité végétale au Sénégal. Science et Changement Planétaires/Sécheresse, 12(3) : 149-55.

Beaugrand G, Goberville E. 2010. Conséquences des changements climatiques en milieu océanique. VertigO-La revue électronique en sciences de l'environnement, $8: 14$. DOI : https://id.erudit.org/iderudit/045530ar

Cazalet B. 2004. Les aires marines protégées à l'épreuve du sous-développement en Afrique de l'Ouest. VertigO-la revue électronique en sciences de l'environnement, 5(3): $16 . \quad$ DOI : https://doi.org/10.4000/vertigo.3274

Compère $\mathrm{P}$, Riaux-Gobain C. 2009. Diatomées de quelques biotopes marins, saumâtres et dulçaquicoles de Guinée (Afrique occidentale). Systematics and Geography 
of Plants, 79(1): 33-66. DOI : https://www.jstor.org/stable/20649771

Dia A. 1983. Biomasse et biologie du phytoplancton le long de la Petite CÔTE Sénégalaise : relation avec l'hydrologie. Archive Scientifique du Crodt du Centre de Recherche Océanographiques de Dakar-Thiaroye, 126 : 77.

De Morais LT, Simier M, Raffray J, Sadio O. 2007. Suivi biologique des peuplements de poissons d'une aire protégée en zone de mangrove : le bolon de Bamboung (Sine Saloum, Sénégal). Institut de Recherche pour le Développement. Dakar, IRD. P.23.

Ecoutin, JM, Sadio O, Simier M, Raffray J, Tito de Morais L. 2012. Comparaison des peuplements de poissons d'une aire protégée en zone de mangrove (le bolon de Bamboung, Sine Saloum, Sénégal) avec les peuplements de deux sites proches non protégés de l'exploitation halieutique. Rapport du Contrat $\mathrm{n}^{\circ}$ CSRP/AFD/C11/2011. Dakar, juin 2020, $67 \mathrm{pp}$.

Gueye M, Ba N, Bodian MY, Mbaye MS, Bassene C, Kane M, Noba K, Ngom, A. 2014. Les Cyanophyceae marines de l'île de Ngor (Sénégal). Int. J. Biol. Chem. Sci., 8(1) :

144-156.

DOI: 10.4314/ijbcs.v8i1.14.

Gueye 2016. Contribution à la connaissance de la diversité et la dynamique spatiotemporelle des cyanophytes et microalgues marines de la presqu'île du Cap Vert et de la petite Côte (Sénégal). Thèse de Doctorat, Université Cheikh Anta Diop, Sénégal, P.232.

Jones GJ, Poplawski W. 1998. Understanding and management of cyanobacterial blooms in sub-tropical reservoirs of Queensland, Australia. Water Science and Technology, 37(2): 161-168. DOI : https://doi.org/10.1016/S0273-

1223(98)00020-1.

Konan ES, Komeo K, Salla M. 2018. Les Diatomées centriques de la lagune de Fresco Côte d'Ivoire. Agronomie Africaine. $\quad 30(2)$ : 179-191. DOI : https://www.ajol.info/index.php/aga/artic le/view/179637.

Ndour I, Berraho A, Fall M, Ettahiri O, Sambe B. 2018. Composition, distribution and abondance of zooplancton and ichthyplankton along the SenegalGuninea maritime zone (West africa). The Egyptian of Aquatic Research, 44(2): 109-124.

DOI : https://doi.org/10.1016/j.ejar.2018.04.00 1.

Paulmier G. 1994. Les dinophycées pélagiques et benthiques du Golfe de Gascogne sud de la Bretagne à Arcachon. Annales de la Société des Sciences Naturelles de la Charente-Maritime, 8(3): 289-357. DOI : https://archimer.ifremer.fr/doc/00260/37 097/.

R Core Team. 2018. R: A language and environment for statistical computing. $\mathrm{R}$ Foundation for Statistical Computing, Vienna, Austria. URL https://www.Rproject.org/.

Sadio O. 2015. Evaluation de l'efficacité des Aires Marines Protégées comme outil de restauration des ressources marines et de gestion des stocks halieutiques : l'expérience ouest africaine. Thèse de Doctorat. Brest. P.217. 